\title{
La Destrucción Creativa y el Desarrollo Económico
}

\section{Creative Destruction and Economic Development}

\author{
Crnl. E.M.C. HUGO RUIZ VILLACRÉS, PH.D. \\ Universidad de las Fuerzas Armadas ESPE, Av. General Rumiñahui s/n y Ambato, Sangolquí - Ecuador
} hdruiz@espe.edu.ec

\begin{abstract}
Varios autores e investigadores en este campo consideran que el fortalecimiento y afinamiento de los sistemas de innovación regionales y nacionales es una importante estrategia para influir positivamente en el crecimiento económico y consecuentemente elevar los estándares de vida de sus habitantes y de la sociedad en general. Debido a sus beneficios económicos incuestionables existe una carrera permanente entre los países que generan tecnología de vanguardia, para llegar primeros a los mercados globales con productos innovadores. Cada vez son más los gobiernos que intentan unirse a la estrategia de impulsar su crecimiento económico mediante la comercialización de nuevos productos tecnológicos resultantes del los procesos de investigación científica, lo que se conoce como economía basada en el conocimiento.
\end{abstract}

Países de Asia, entre otros en el resto del mundo, han invertido de forma sostenida en el desarrollo de sus sistemas de innovación tecnológica, en la modernización y actualización de las instituciones dedicadas a la investigación, destinan más fondos para fortalecer los procesos de investigación científica con la intención de que estas iniciativas impulsen el crecimiento de los sectores industriales, aceleren la sofisticación de sus procesos de manufactura, atraigan inversión extranjera directa y como resultado establezcan en el mediano plazo los cimientos para una economía basada en el conocimiento.

Estos países dedican esfuerzos para mejorar la colaboración entre la industria y la universidad, establecen un marco legal adecuado que fomente el respeto a los derechos de propiedad intelectual, que inspire la confianza y tranquilidad a los inversionistas foráneos, creando estructuras institucionales complejas e interrelacionadas que impulsen el emprendimiento y les tornen más competitivos en los mercados globalizados.

La pregunta que necesariamente deben hacerse los países que optan por esta estrategia es: ¿̇cuáles son los factores básicos que deben estar presentes en un ecosistema para que el proceso de innovación científica tenga éxito? La respuesta no es sencilla y 
de hecho no existe una sola respuesta, todo depende: En primer lugar del entorno y de las condiciones circundantes en cada caso, pero cuando se habla de ecosistemas de innovación, existen aquellos factores que deben estar presentes obligatoriamente, independientemente de las características geográficas o de diferencias políticas e ideológicas.

Un ecosistema de innovación científica y tecnológica debe contar con varios factores que obligatoriamente deben coexistir y en las proporciones adecuadas para contribuir con el equilibrio del sistema, de ahí el nombre de ecosistema. La falta o exceso de uno, provocaría un desbalance que puede resultar perjudicial para otro u otros componentes funcionales del sistema. Por el contrario, estos elementos al estar presentes y correctamente balanceados, van a permitir que las ideas y nuevos descubrimientos, sea que provengan de procesos de investigación en las industrias del sector privado o de los resultados del trabajo científico en los laboratorios de las universidades, completen el ciclo de innovación y terminen con productos que se comercialicen y sean adquiridos por una sociedad que genera demanda, que exige cada vez mejores productos, más modernos, que mejoren su calidad de vida y aumenten su comodidad y satisfacción.

A este proceso innovativo, Joseph Schumpeter lo definió como "la destrucción creativa". De acuerdo a Schumpeter, la innovación es el desplazamiento o suplantación de los productos que la sociedad está consumiendo actualmente, por otros, concebidos como resultado del dominio y desarrollo de nuevo conocimiento, de tecnologías disruptivas, con mejores características de durabilidad, métodos de fabricación, precio o calidad y que en consecuencia superan a sus antecesores, haciendo que sean adoptados rápidamente por los consumidores, causando como consecuencia inevitable que los productos de tecnología obsoleta, agoten su demanda y salgan del mercado, ocasionando su fin o destrucción, por efecto de la creatividad científica.

El mundo está cada vez más expuesto a la competencia tecnológica, lo que se evidencia en la implementación de políticas públicas que impulsan la innovación, tales como el desarrollo de las capacidades e incentivos para las pequeñas y medianas empresas, la asignación de mayores presupuestos nacionales destinados para la investigación y el desarrollo, el fortalecimiento de los sistemas de educación superior y la modernización y mejoramiento de institutos públicos de investigación.

Gobiernos como los de Francia, Gran Bretaña, Finlandia, Holanda, Estados Unidos, China, Korea del Sur y Japón, por mencionar solo algunos, incentivan y posibilitan la creación de clusters tecnológicos donde se agrupen empresas e industrias afines con una meta común: el desarrollo y la comercialización de nuevas tecnologías. Históricamente se ha evidenciado que los resultados de estas iniciativas no son visibles de inmediato, sino que son iniciativas que maduran en el mediano y largo plazo, luego de que han pasado décadas desde su implementación, por lo que existen dudas sobre la efectividad que tendrían estas iniciativas, si son adoptadas e implementadas por otros países del mundo.

Dentro de los sistemas de innovación regionales y nacionales exitosos se evidencia que la incubación de empresas con tecnologías emergentes, se constituyen en generadores de plazas de trabajo y son catalizadores del crecimiento económico. Muchos estudios se han realizado acerca 
de cuáles son los factores que favorecen la innovación científica y tecnológica, pero siendo un proceso complejo, el éxito no sucede espontáneamente. No son suficiente los factores elementales y básicos, se require también de su interacción apropiada y coordinada para que los resultados afloren.

Los investigadores que han intentado definir cuales serían aquellos factores que favorecen y posibilitan la innovación tecnológica se han enfocado en su gran mayoría a buscar los resultados de modelos de innovación en los países desarrollados: Estados Unidos, Alemania, Bélgica, Reino Unido, España, y varios otros países tanto de Asia como de Europa, muchos de los datos estadísticos que existen para poder evaluar estos sistemas, se refieren a los países miembros de la OECD. Lamentablemente, muy pocos estudios hacen referencia a los resultados de estas iniciativas de innovación tecnológica en países en vías de desarrollo, sea porque el número de proyectos es limitado - porque los resultados de estas iniciativas aún no se han hecho evidentes y por lo tanto cuantificables.

Con excepción de la China e India, y pocos casos de Brazil, Chile y México, la efectividad de estos procesos y su aplicabilidad para los países en vías de desarrollo, todavía es incierta, los resultados no se concretan, por tratarse de procesos que tardan en arraigarse y consolidarse, y que por lo tanto demandan varios años en rendir sus frutos.

No se tenía evidencia contundente acerca de cuales serían las implicaciones para una nación en vías de desarrollo dispuesta a incursionar y seguir por el camino de la economía basada en el conocimiento, ¿̇qué factores deben de tomarse en cuenta como mínimo para arrancar?, ¿̇cuáles son las condiciones básicas para garantizar que los resultados de las ingentes inversiones nacionales sean prometedoras?. ¿Podría el éxito, que hasta hoy ha sido un privilegio solamente de los países desarrollados, trasladarse a los países que emprenden con estas iniciativas en otras regiones del mundo? La intención es tratar de emular el éxito de Silicon Valley, localmente. La brecha tecnológica entre los países que innovan y los que han tardado en adoptar estas estrategias de emprendimiento tecnológico se sigue ampliando y como consecuencia inevitable, también la brecha económica y de la calidad de vida de sus habitantes.

Hay incertidumbre en los países en desarrollo acerca de los componentes básicos necesarios y otros factores que se necesitan tener en marcha de modo que estas inversiones nacionales traigan resultados favorables. Las lecciones aprendidas y enseñanzas de países exitosos que cuentan con una considerable experiencia en la implementación y el crecimiento de sus sistemas de innovación tecnológica, bien podrían ser aplicables a nuevas iniciativas en otros países, que recién incursionan en esta estrategia.

Es importante conocer cuales son estos componentes básicos, que sin importar la ubicación geográfica, cultura, o tendencia política, son comunes y básicos para todos, además de como éstos están interrelacionados. Esta información, va a permitir que los planificadores, gobernantes e impulsadores de nuevos proyectos comprendan el proceso de innovación tecnológica en su contexto y tomen los pasos necesarios y correctos cuando diseñen o implementen nuevos sistemas de innovación. 
Los países que quieren dar el giro de la economía tradicional basada en la disponibilidad y explotación de recursos naturales hacia la economía del conocimiento que proviene de los resultados de la investigación científica, deben tener especificidades o condiciones de partida que han de implementarse antes de importar iniciativas tecnológicas del mundo desarrollado. Existen características propias, que podrían ser decisivas y que pueden permitir o no, que estos nuevos proyectos maduren para que sean eficientes y efectivos.

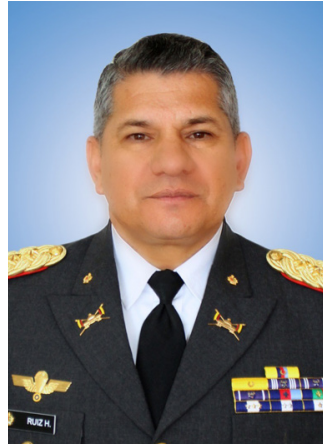

El coronel HUGO RUIZ VILLACRÉS obtuvo su Ph.D en Tecnología en Purdue University en los Estados Unidos de Norteamérica; es Ingeniero mecánico de la Escuela Politécnica del Ejército; además, ha realizado estudios de posgrado en Middle Tennessee State University y en State University of New York at Buffalo. Recibió la beca Fulbright en el Faculty Development Program año 2010.

Su formación doctoral le permite la propuesta y administración de proyectos de investigación científica de gran escala, tanto en el sector público como privado, y en el diseño y ejecución de proyectos de investigación con una aproximación tanto cuantitativa, como cualitativa o dual. Los cursos de formación de posgrado más relevantes son Robotics, Production Systems Engineering, Computer Integrated Manufacturing, Qualitative Research, Research Design, Nanotechnology.

Sus temas de interés en el campo de la investigación son el desarrollo tecnológico desde un enfoque multidisciplinario y un particular interés en la intersección entre la innovación científica y el desarrollo tanto local, regional y nacional. $\mathrm{Ha}$ sido expositor en varios congresos internacionales, y docente de diversas asignaturas. Ha ocupado varios cargos en la ESPE, los más relevantes: director del Centro de Mecatrónica, decano de Ingeniería Mecánica, director de la ESPE Latacunga, vicerrector de Docencia, y el último cargo desempeñado es el de vicerrector Académico General. Actualmente, tiene el grado de coronel y ocupa el cargo de director de Comunicación Social del Ejército Ecuatoriano. 